\title{
Design Consideration and Impact of Gate Length Variation on Junctionless Strained Double Gate MOSFET
}

\author{
K. E. Kaharudin, Ameer F. Roslan, F. Salehuddin, Z. A. F. M. Napiah, A. S. M. Zain
}

\begin{abstract}
Aggressive scaling of Metal-oxide-semiconductor Field Effect Transistors (MOSFET) have been conducted over the past several decades and now is becoming more intricate due to its scaling limit and short channel effects (SCE). To overcome this adversity, a lot of new transistor structures have been proposed, including multi gate structure, high-k/metal gate stack, strained channel, fully-depleted body and junctionless configuration. This paper describes a comprehensive 2-D simulation design of a proposed transistor that employs all the aforementioned structures, named as Junctionless Strained Double Gate MOSFETS (JLSDGM). Variation in critical design parameter such as gate length $\left(L_{g}\right)$ is considered and its impact on the output properties is comprehensively investigated. The results shows that the variation in gate length $\left(L_{g}\right)$ does contributes a significant impact on the drain current $\left(I_{D}\right)$, on-current $\left(I_{O N}\right)$, off-current (I $\left.I_{\text {OFF }}\right), I_{O N} / I_{\text {OFF }}$ ratio, subthreshold swing (SS) and transconductance $\left(g_{m}\right)$. The JLSDGM device with the least investigated gate length (4nm) still provides remarkable device properties in which both $I_{O N}$ and $g_{m}(\max )$ are measured at 1680 $\mu \mathrm{A} / \mu \mathrm{m}$ and $2.79 \mathrm{mS} / \mu \mathrm{m}$ respectively.
\end{abstract}

Keywords : Gate length, on-current, off-current, subthreshold swing, transconductance.

\section{INTRODUCTION}

$\mathrm{O}$ ver the past decades, the dimension of a transistor has been miniaturized from micrometer into nanometer regime, following the Moore's law prediction. Scaling attempt in a transistor's size has been proven to increase the drive current and also the device's speed. However, transistor's scaling is becoming even more intricate with each successive transistor generation reaches the theoretical and technological restriction. For instance, the aggressive scaling of a transistor reaches a critical situation where an extremely thin gate oxide are no longer capable of suppressing the short channel effects (SCE) effectively [1]. As a result, the SCE is becoming more severe, thus degrading both gate controllability and electron mobility of a transistor [2].

High leakage current is regarded as one of the main obstacles in transistor's scaling. The traditional $\mathrm{Poly} / \mathrm{SiO}_{2}$ stack structure is no longer practical for sub-nanometer

Revised Manuscript Received on July 22, 2019

* Correspondence Author

K. E. Kaharudin, Z. A. F. M. Napiah, F. Salehuddin*, Ameer F. Roslan, A. S. M. Zain, MiNE, Centre for Telecommunication Research and Innovation (CeTRI), Faculty of Electronics and Computer Engineering, Universiti Teknikal Malaysia Melaka, Hang Tuah Jaya, 76100 Durian Tunggal, Melaka.

Email: khairilezwan@yahoo.com.my, fauziyah@utem.edu.my. transistor technology mainly due to SCE and poly depletion effects. An ultra-small transistor requires an increased capacitance of gate dielectric to suppress SCE problems, which can be achieved by reducing the insulator's thickness [3]. However, a massive attempt on reducing the gate oxide would results in larger gate leakage, which consequently affects the transistor's performances. Theoretically, the leakage current is found unacceptably high when the gate oxide is scaled below $2 \mathrm{~nm}$ of thickness [4]. As a solution, an increased dielectric material (high- $k$ ) is employed to substitute silicon dioxide $\left(\mathrm{SiO}_{2}\right)$ in which a thicker dielectric layer can be growth on the top of silicon body without having electrical thickness penalties [5]. The high- $k$ dielectric is normally incorporated with metal-gate for solving the compatibility issue between poly-gate and high- $k$ dielectric that might result in lower on-current $\left(I_{O N}\right)[6]$.

Multi-gate transistor is one of the solutions to prolong the continuity of transistor scaling. This type of structure takes advantage of an enhance channel to reduce the SCE. The term "multi-gate" refer to the transistor's structure that comprises more than a single gate in which these gate electrodes is wrapped around the channel region [7]. One of the common configurations of multi-gate transistor is called double-gate MOSFET. Double-gate MOSFET's structure has been proven to be less sensitive to SCE mainly because of its excellent electrostatic control over the channel region [8-11]. The mobility of the electrons/holes within the conductive channel are improved due to its excellent gate control that allows a significant amount of volume inversion [12]. The current drivability in the double-gate structure can be improved without having any increment in the area by simply etching the silicon body a lot more thinner[13, 14].

Fully depleted configuration is an important feature used in most of double-gate and silicon-on-insulator (SOI) transistors [15-20]. The fully depleted channel is found to be very effective in mitigating the SCE [21-23]. The SCE become more prominent when the subthreshold swing (SS) is increased in which the degrading drain current $\left(I_{D}\right)$ stands up against a gate voltage $\left(\mathrm{V}_{\mathrm{G}}\right)$ and threshold roll-off to negative direction in the case of the $n$-channel transistor. These effects should be well mitigated by lessening the thickness of silicon body to less than $20 \mathrm{~nm}$ [24]. In ultrathin channel, the majority carriers (electrons/holes) concentration will be fully depleted when a certain amount of voltage is supplied to the gate terminal. 
As a certain threshold reached, the channel begins to deplete and a surface inversion layer is formed. The fully depleted channel could avoid high electric field that might cause an increased SS at high drain bias, thanks to a fully or partly elimination of floating body effect [25-27].

Strained silicon is also one of the alternative methods to improve the carrier mobility of a transistor [28-30]. The strained silicon method offers significant enhancements in transistor's performance via tuning the material properties rather than geometrical structure and carrier's density [31]. The physical attributes of strained silicon is basically due to the presence of incorporated germanium in the silicon substrate [32]. As the different layers stacking on each other, the strained channel would experience a change in band gap energies [33]. The primary advantage of strained silicon method is to form high mobility area within the conductive channel region. However, the mobility within strained channel is often diminished by phonon scattering as the electrons/holes are not totally restrained due high electric field [34]. As a solution, high doping concentrations and shorter channel are employed so that the carrier mobility can be effectively enhanced.

Junctionless configuration is another alternative solution to allow the continuity of transistor's shrinkage without degrading the device performance [35-38]. This configuration has been aggressively studied and applied by many researchers due to its simplified fabrication process [39]. Junctionless transistor totally neglects the need of complicated channel and source/drain S/D engineering in forming an ultra-shallow S/D junctions with high doping concentration gradient [40]. The working principle of junctionless transistor is totally relies on the heavily doped channel and S/D regions that utilizes similar type of dopants [41]. Junctionless transistor is normally fabricated using thin/narrow semi conductive layer which enables the majority carriers to be fully depleted during off-state condition [42]. Highly doped of an extremely thin channel would allow a decent flow of current from the source to the drain as the transistor is switched on [43].

This paper focuses on describing a comprehensive 2-D simulation design of a new transistor's structure with consideration of all the aforementioned methods, called as Junctionless Strained Double Gate MOSFETs (JLSDGM). Furthermore, the impact of critical parameter variations on the output properties is also investigated. The organization of this work is structured as follows: Section 2 describe the device structure of JLSDGM device and its process flow using Silvaco Athena TCAD tools. Section 3 briefly explains the transistor's simulation employing Silvaco Atlas device simulator. Section 4 provides a comprehensive insight on the impact of gate length $\left(L_{g}\right)$ on drain current $\left(I_{D}\right)$, on-current $\left(I_{O N}\right)$, off-current $\left(I_{O F F}\right), I_{O N} / I_{O F F}$ ratio, subthreshold swing $(S S)$ and transconductance $\left(g_{m}\right)$. Section 5 suggests the possible recommendations for the future work. The conclusion summarizing this simulation study is presented later in section 6.

\section{PROCEDURE FOR PAPER SUBMISSION}

2-D process simulation for the JLSDGMs was conducted in which the process flow of the device is shown in Fig.1. The process simulation (via Athena module) comprises the initial SiGe substrate $(\mathrm{Ge}<20 \%)$, ultrathin silicon layer formation $(1 \mathrm{~nm})$, channel doping, high-k/metal-gate (HKMG) deposition, source/drain doping, metallization and structure reflection.

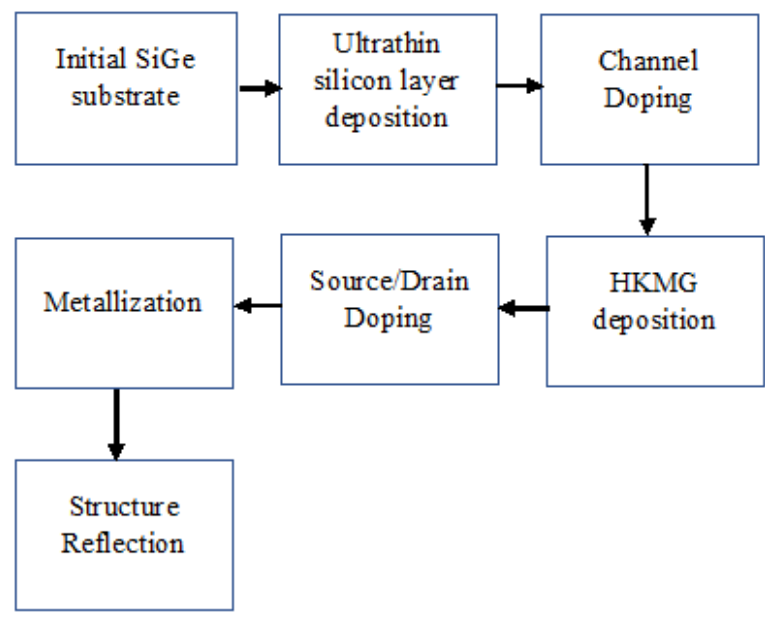

Fig. 1. Simulated process flow for ultra-thin JLSDGM

The structure of JLSDGM device employs silicon germanium ( $\mathrm{SiGe}$ ) with thickness of $8 \mathrm{~nm}$ as the main substrate due to its larger interatomic distances compared to a pure crystal of silicon. Then, an ultrathin silicon film is formed on the top of SiGe layer in order to create a strained silicon layer. The strained silicon layer is formed due to a phenomenon in which the silicon atoms are strongly stretched from each other as the majority atoms inside the conductive channel attempting to align with the $\mathrm{SiGe}$ atoms. As a result, the ultrathin silicon layer becomes tensile stretched substantially due to its relatively smaller lattice constant than the main substrate ( $\mathrm{SiGe}$ ). The strained channel is then heavily doped with $1 \times 10^{17} \mathrm{~cm}^{-3}$ of Arsenic dose (n-type). Double-gate configuration is applied in order to extend enhanced gate controllability over the channel. Tungsten silicide $\left(\mathrm{WSi}_{\mathrm{x}}\right.$ ) layer is used as the metal-gate due to its tunable work function capability [44]. This $\mathrm{WSi}_{\mathrm{x}}$-gate is then deposited on a high- $k$ dielectric layer which provides better compatibility than a poly-gate.

Titanium dioxide $\left(\mathrm{TiO}_{2}\right)$ is used as a gate dielectric attributable to its remarkable high permittivity $(\sim 85 \mathrm{eV})$ which allows the employment of thicker insulator. For instance, a $\mathrm{TiO}_{2}$ layer with a dielectric constant of $85 \mathrm{eV}$ (compared to $3.9 \mathrm{eV}$ for silicon dioxide) can be deposited 21 times thicker than silicon dioxide $\left(\mathrm{SiO}_{2}\right)$ which subsequently assists in reducing the leakage of electrons across the dielectric layer, while retaining the similar capacitance [45]. Therefore, equivalent oxide thickness (EOT) is used to indicate how thick $\mathrm{SiO}_{2}$ layer would need to be to produce the same effect as the $\mathrm{TiO}_{2}$ material being used. Since the thickness of $\mathrm{TiO}_{2}$ used in this structure is $3 \mathrm{~nm}$, the EOT can be calculated as: 


$$
E O T=\left[\frac{\varepsilon_{\mathrm{SiO} 2}}{\varepsilon_{\mathrm{TiO} 2}}\right] T_{\mathrm{TiO} 2}
$$

where $\varepsilon_{\mathrm{SiO} 2}$ is the permittivity of $\mathrm{SiO}_{2}$ which is $3.9, \varepsilon_{T i O 2}$ is the permittivity of $\mathrm{TiO}_{2}$ which is 85 and $T_{T i O 2}$ is the physical thickness of $\mathrm{TiO}_{2}$ layer. Hence, the EOT of the insulator for this structure would be $0.138 \mathrm{~nm}$.

The source/drain (S/D) regions for n-type device are doped with the same type of dopant used in previous channel doping process in order to form $\mathrm{N} \mathrm{N}^{+} \mathrm{N}$ configuration (junctionless). The metallization process is then conducted by sputtering the aluminum layer on the top part of the device. Any excessive aluminum layer is removed accordingly to pattern the contacts and the electrodes. The investigated geometrical and process parameters of the JLSDGMs are listed in Table I. Finally, the structure is reflected in both $x$ and $y$ directions as depicted in Fig. 2.

Table- I: Parameters used in the simulated JLSDGMs

\begin{tabular}{|c|c|c|c|}
\hline Variables & Units & $\begin{array}{c}\text { Fixed } \\
\text { value }\end{array}$ & $\begin{array}{c}\text { Manipulate } \\
\mathrm{d} \\
\text { range }\end{array}$ \\
\hline Gate Length, $L_{g}$ & $\mathrm{~nm}$ & 6 & $4-12$ \\
\hline Channel doping, $N_{c h}$ & $\begin{array}{c}\text { Atom/cm } \\
3\end{array}$ & $1.0 \mathrm{E} 17$ & N/A \\
\hline $\begin{array}{c}\text { S/D doping, } N_{s d} \\
\text { Atom/cm } \\
3\end{array}$ & $1.0 \mathrm{E} 13$ & N/A \\
\hline $\begin{array}{c}\text { Metal work-function, } \\
W F\end{array}$ & $\mathrm{eV}$ & 4.6 & N/A \\
\hline
\end{tabular}

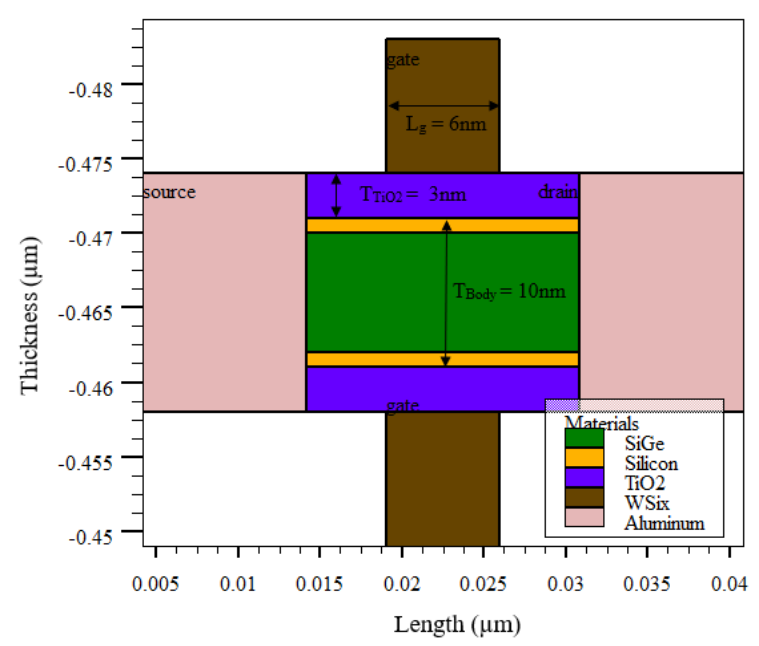

Fig. 2. 2-D cross section of the JLSDGM device

\section{DEVICE SIMULATION}

Device simulation for n-type JLSDGM device is carried out through Silvaco TCAD Atlas module. The output structure from the previous Athena module is fed into Atlas module as mesh infile. Fig. 3 depicts the contour mode of the JLSDGM device, showing the net doping for SiGe, silicon, titanium dioxide, tungsten silicide and aluminum. Based on the contour mode, the effective channel length $\left(L_{e f f}\right)$ is approximately measured at $8 \mathrm{~nm}$. device simulation employs a built-in physical model that takes the drift diffusion (DD) model, simplified Boltzmann carrier
The n-type JLSDGM device is characterized via device simulation (Atlas module) in linear and saturation mode. The

statistics, inversion mobility model combined with Shockley-Read-Hall (SRH) recombination into account. Since the proposed device is non-planar, the Lombardi CVT model is selected to provide an accurate simulation [46]. The Lombardi CVT model is employed for considering the scattering processes such as lattice vibration (phonons), impurity ions, surfaces, and other material imperfections during the simulation. Critical device characteristics such as on-state current $\left(I_{O N}\right)$, off-state current $\left(I_{O F F}\right), I_{O N} / I_{O F F}$ ratio, subthreshold swing $(S S)$ and transconductance $\left(g_{m}\right)$ can be extracted from the generated $I_{D^{-}} V_{G}$ curve. The device simulation conditions for the investigated characteristics are set up as shown in Table II [46].

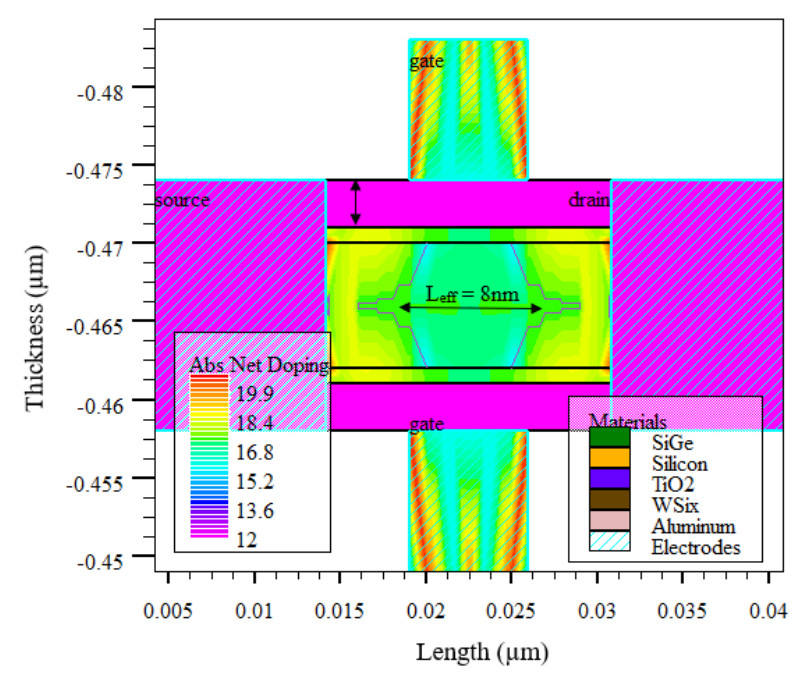

Fig. 3. Contour mode of the JLSDGM device

Table- II: Device Simulation Conditions

\begin{tabular}{|l|c|c|c|c|}
\hline Electrical & Drain Voltage, & \multicolumn{3}{|c|}{ Gate Voltage, $\mathrm{V}_{\mathrm{G}}(\mathrm{V})$} \\
\cline { 3 - 5 } Characteristics & $\mathrm{V}_{\mathrm{D}}(\mathrm{V})$ & $\mathrm{V}_{\text {Initial }}$ & $\mathrm{V}_{\text {Step }}$ & $\mathrm{V}_{\text {Final }}$ \\
\hline $\begin{array}{l}\text { Threshold Voltage, } \\
\mathrm{V}_{\mathrm{TH}}(\mathrm{V})\end{array}$ & 0.5 & 0 & 0.1 & 1.0 \\
\hline $\begin{array}{l}\text { On-state Current, } \\
\mathrm{I}_{\mathrm{ON}}(\mu \mathrm{A} / \mu \mathrm{m})\end{array}$ & 0.5 & 0 & 0.1 & 1.0 \\
\hline $\begin{array}{l}\text { Off-state Current, } \\
\text { IOFF }(\mathrm{A} / \mu \mathrm{m})\end{array}$ & 0.5 & 0 & 0.1 & 1.0 \\
\hline $\begin{array}{l}\text { Subthreshold } \\
\text { Slope, SS } \\
(\mathrm{mV} / \text { decade) }\end{array}$ & 0.5 & 0 & 0.1 & 1.0 \\
\hline $\begin{array}{l}\text { Transconductance, } \\
\mathrm{gm}_{\mathrm{m}}(\mathrm{mS} / \mu \mathrm{m})\end{array}$ & 0.5 & 0 & 0.1 & 1.0 \\
\hline
\end{tabular}

\section{IMPACT OF GATE LENGTH VARIATIONS ON JLSDGM'S PERFORMANCES}

For the purpose of comprehensive invesigation on the behaviors of JLSDGM device, the impact of the gate length variations on $I_{D}-V_{G}$ transfer characteristics is presented in this section. Section A, B, C, and D describe the impact of variations in gate length $\left(L_{g}\right)$ upon the $I_{O N}, I_{O F F}, I_{O N} / I_{O F F}$ ratio, $S S$ and $g_{m}$ respectively.

\section{A. Impact of Gate Length Variations on On-current}

In transistor's fabrication, physical gate length $\left(L_{g}\right)$ is the most crucial parameter which is grown using the minimum feature size of mask in a particular technology node. 
The impact of $L_{g}$ variations on the device's characteristics is important to be investigated because it could contribute significant changes in $L_{e f f}$ as well as the saturation current. Fig. 4 shows the combined plot of $I_{D^{-}} V_{G}$ transfer characteristics at $V_{D}=0.5 \mathrm{~V}$ in linear and $\log$ scales for multiple $L_{g}$ variations.

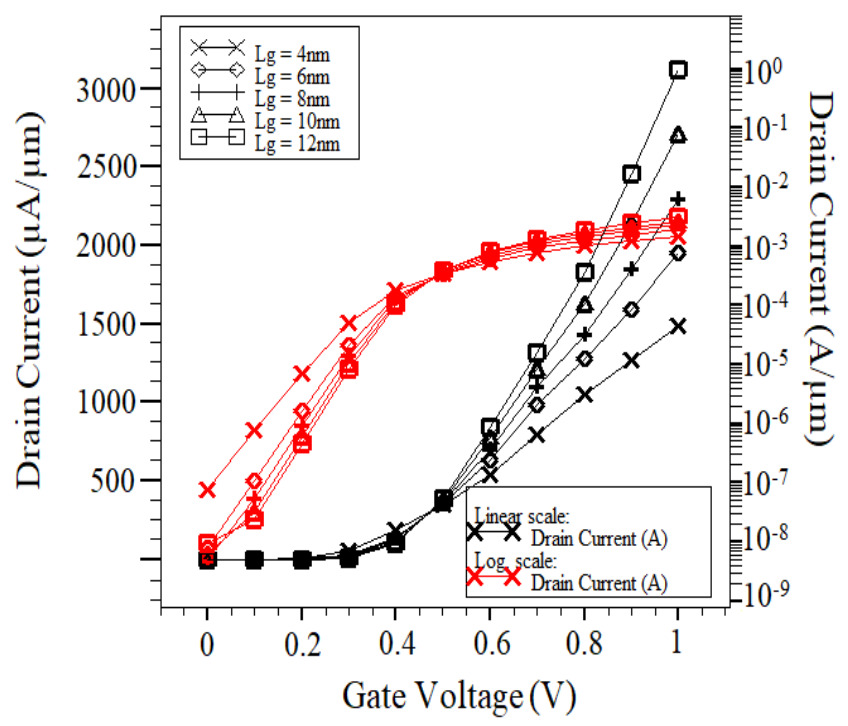

Fig. 4. Combined plot of the $\mathrm{I}_{\mathrm{D}^{-}}-\mathrm{V}_{\mathrm{G}}$ transfer characteristics at $\mathrm{V}_{\mathrm{D}}=0.5 \mathrm{~V}$

It is observed that the drain current $\left(I_{D}\right)$ of the JLSDGM device is gradually decreased as the $L_{g}$ is scaled down from $12 \mathrm{~nm}$ to $4 \mathrm{~nm}$, shifting the $I_{D^{-}} V_{G}$ curves towards the maximum positive $x$-axis $(1 \mathrm{~V})$. Shrinking the $L_{g}$ would reduce the area of the inverted channel, predominantly due to the shorter distance between the source and the drain regions. As a result, the $V_{T H}$ decreases, causing the $I_{D}$ to gradually decrease as the $L_{g}$ is further scaled down. However, the maximum $I_{D}$ at $V_{G}=$ $1 \mathrm{~V}$, also known as on-current $\left(I_{O N}\right)_{-}$is still considerably high for the device with $4 \mathrm{~nm}$ of gate length, thanks to the positive impact of double-gate structure, ultrathin body, strained channel and junctionless configuration. Fig. 5 depicts the impact of $L_{g}$ variations on the $I_{O N}$ characteristic of the JLSDGM device.

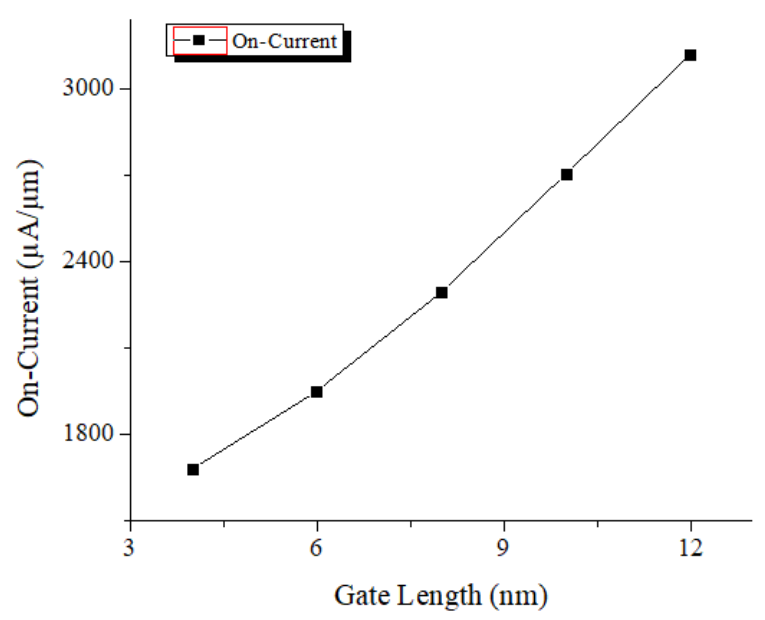

Fig. 5. Plot of on-current versus gate length

Based on the plot, it is observed that the $I_{O N}$ increases almost linearly with the increase of the $L_{g}$. The $I_{O N}$ of the JLSDGM device is decreased by approximately $46 \%$ as the $L_{g}$ is scaled down from $12 \mathrm{~nm}$ to $4 \mathrm{~nm}$. The device with $4 \mathrm{~nm}$ of gate length still exhibits a remarkable on-current characteristic which is measured at $1680 \mu \mathrm{A} / \mu \mathrm{m}$. This is mainly contributed from the implementation of double-gate structure and HKMG that boost the amount of electron inversion into the channel region, even with a shorter effective channel length. The double-gate mode enhances the ability to regulate the distribution of the field and flow of the current in the channel. With an extra gate, the gate to channel coupling is doubled, resulting in a better SCE's suppression and higher current density [47].

The utilization of HKMG also contributes to high $I_{O N}$ value due to a total elimination of poly depletion effects. The JLSDGM device with HKMG emulates like it has a thinner oxide in inversion with associated improved capacitance that subsequently results in significant $I_{O N}$ improvements. Furthermore, the reduction in $L_{g}$ cause high average stress across the channel due to much smaller channel region [48]. The application of strained channel is aimed to boost the electron mobility with high doping concentration, thus leading to higher on-current [49].

\section{B. Impact of Gate Length Variation on Off-current}

According to Fig. 4, the off-current $\left(I_{O F F}\right)$ is measured when the gate voltage $\left(V_{G}\right)$ is zero and the drain voltage $\left(V_{D}\right)$ is set equal to the drain supply $\left(V_{D D}\right)$ which is $0.5 \mathrm{~V}$. In other words, $\mathrm{I}_{\mathrm{OFF}}$ is the drain current $\left(I_{D}\right)$ when there is no input voltage supplied to the gate. As the gate length shrinks, $\mathrm{I}_{\mathrm{OFF}}$ becomes one of the crucial characteristics that need to be considerably monitored. Fig. 6 depicts the impact of $L_{g}$ variation on $I_{O F F}$ characteristic.

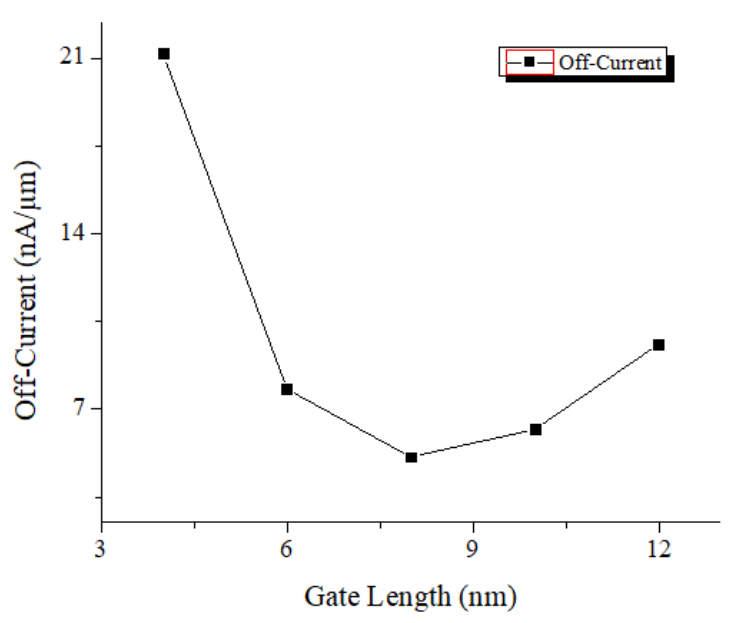

Fig. 6. Plot of off-current versus gate length

Based on the plot, it can be concluded that the $L_{g}$ variation cause unpredictable behavior of drain current in off-state condition. Such occurrence is mainly due to the variation of physical gate length upon the band-to-band-tunneling (BTBT) current. During off-state condition, the electrons in the channel is randomly distributed that result in uncertain amount of positive trapped charges in the insulator. This would lead to random variations within the electric field eventually causing erratic leakage behaviors. 
The JLSDGM device with $4 \mathrm{~nm}$ of $L_{g}$ demonstrates the highest $I_{O F F}$ value, implying that the channel begin to suffer from the SCE at this point.

\section{Impact of Gate Length Variations on $\mathbf{I}_{\mathrm{ON}} / \mathbf{I}_{\mathrm{OFF}}$ ratio}

$I_{O N} / I_{O F F}$ ratio is the numerical expression to indicate increased performance (higher $I_{O N}$ ) and reduced leakage power (lower $I_{O F F}$ ) for the CMOS transistors. Normally, when a transistor has more gate control over the channel, the $\mathrm{I}_{\mathrm{ON}} / \mathrm{I}_{\mathrm{OFF}}$ ratio would be larger. The $I_{O N} / I_{O F F}$ ratio in JLSDGM device is largely determined by the position of the $\mathrm{V}_{\mathrm{TH}}$ in which its value would significantly affect the amount of current in on-state and off-state condition. The $I_{O N} / I_{O F F}$ ratio of JLSDGM device is measured at $V_{G}=0 \mathrm{~V}$ and $V_{G}=1 \mathrm{~V}$ while $V_{D}=0.5 \mathrm{~V}$ (constant) and equals to maximum allowed $V_{D D}$, which can be calculated as:

$$
I_{O N} / I_{O F F} \text { ratio }=\frac{I_{O N}\left(I_{D} \text { when } V_{G}=1 V\right)}{I_{O F F}\left(I_{D} \text { when } V_{G}=0 V\right)}
$$

Fig. 7 depicts the impact of $L_{g}$ variation on $I_{O N} / I_{O F F}$ ratio for JLSDGM device.

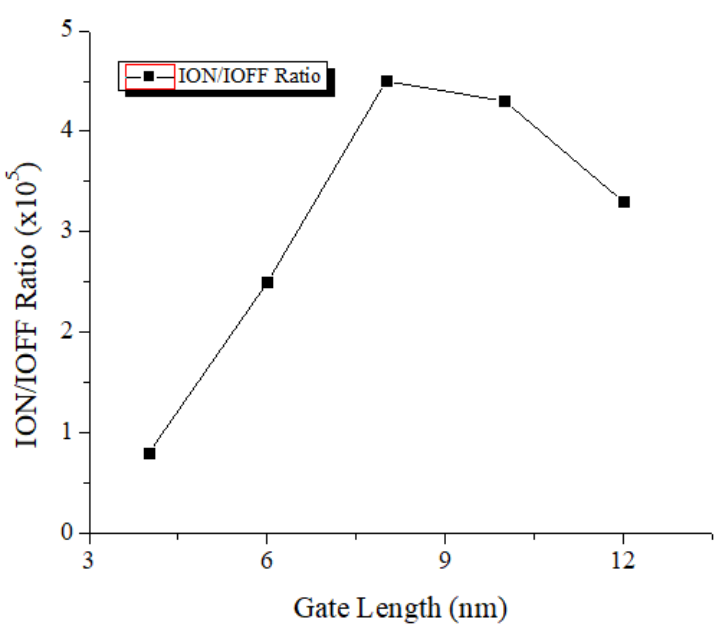

Fig. 7. Plot of $\mathrm{I}_{\mathrm{ON}} / \mathrm{I}_{\mathrm{OFF}}$ ratio versus gate length

Based on the plot, it can be seen that the $I_{O N} / I_{O F F}$ ratio improves linearly as the $L_{g}$ increases from $4 \mathrm{~nm}$ to $8 \mathrm{~nm}$. However, the $I_{O N} / I_{\text {OFF }}$ ratio begins to decline when the $L_{g}$ is scaled beyond $8 \mathrm{~nm}$. This is because the variation in $L_{g}$ has caused slight increases in drain leakage, which makes the device requires a larger gate voltage to turn on. The JLSDGM device with $8 \mathrm{~nm}$ of $L_{g}$ exhibits the largest $I_{O N} / I_{O F F}$ ratio which is measured at $4.5 \times 10^{5}$. The largest $I_{O N} / I_{O F F}$ ratio of a transistor should be attained as large as possible because it decides the speed requirement in logic stage and current carrying capability. The larger $I_{O N} / I_{O F F}$ ratio would contribute to much lower static power dissipation due to off-state leakage. From the perspective of inverter's design consideration which an inverter comprises a series of of two resistors, the larger $I_{O N} / I_{O F F}$ ratio between these two equivalent resistors yields better output level.

\section{Impact of Gate Length Variations on SS}

Subthreshold swing $(S S)$ is a crucial characteristic that decides the scalability limits of a transistor. When the $I_{D}$ is plotted on a logarithmic scale as shown in Fig. 4, the $I_{D}$ is observed varies exponentially with the $\mathrm{V}_{G}$ below the threshold value. The increasing rate of $I_{D}$ below the $V_{T H}$ is characterized by the $S S$, which is relationally calculated as:

$$
S S=\left[\frac{d V_{G}}{d\left(\log _{10} I_{D}\right)}\right]
$$

Fig. 8 depicts the impact of $L_{g}$ variation on $S S$ for JLSDGM device.

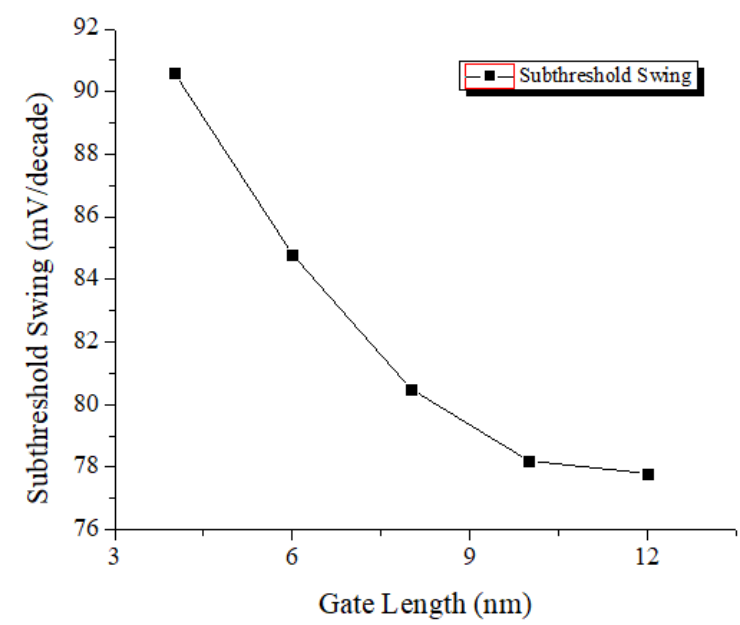

Fig. 8. Plot of subthreshold swing versus gate length

Based on the plot, it can be concluded that the variations in $L_{g}$ is inversely proportional with the $S S$, which means that as the $L_{g}$ is further scaled down from $12 \mathrm{~nm}$ to $4 \mathrm{~nm}$, the SS value is constantly increasing. The $S S$ should be minimized when it is allowed by certain applications. Smaller $S S$ implies that the JLSDGM has better controllability of the gate governing the channel that leads to much improved $I_{O N} / I_{O F F}$ ratio, low leakage and low power consumption. In practice, the gate control is not always perfect due the electrostatic coupling between the channel and the ultrathin body via the depletion layer [7]. The SCE becomes noticeable when there is an increase in $S S$ in which the degrading $I_{D}$ withstands the increasing $V_{G}$ with threshold roll-off to negative direction. These effects can be effectively suppressed by etching the silicon body much thinner, thus subsequently decreasing the $S S$. The JLSDGM device with $12 \mathrm{~nm}$ of $L_{g}$ demonstrates the lowest $S S$ which is measured at $77.8 \mathrm{mV} /$ decade. A lower $S S$ value indicates that the device has a very high-speed switching capability, meaning that less changes in $V_{G}$ is required to increase one decade of $I_{D}$.

\section{E. Impact of Gate Length $\left(\mathrm{L}_{\mathrm{g}}\right)$ Variation on $g_{m}$}

Transconductance $\left(g_{m}\right)$ is a numerical expression that represents the output performance of a transistor, expressing the relation between the current through the output and the voltage across the input. 
The transconductance $\left(g_{m}\right)$ in JLSDGM device is particularly defined as the change in the $I_{D}$ divided by the minor change in the $V_{G}$ with a constant $V_{D}=0.5 \mathrm{~V}$ which is mathematically described as:

$$
g_{m}=\frac{\partial I_{D}}{\partial V_{G}}
$$

Fig. 9 depicts a plot of transconductance $\left(g_{m}\right)$ as a function of gate voltage $\left(V_{G}\right)$ for multiple $L_{g}$ variation.

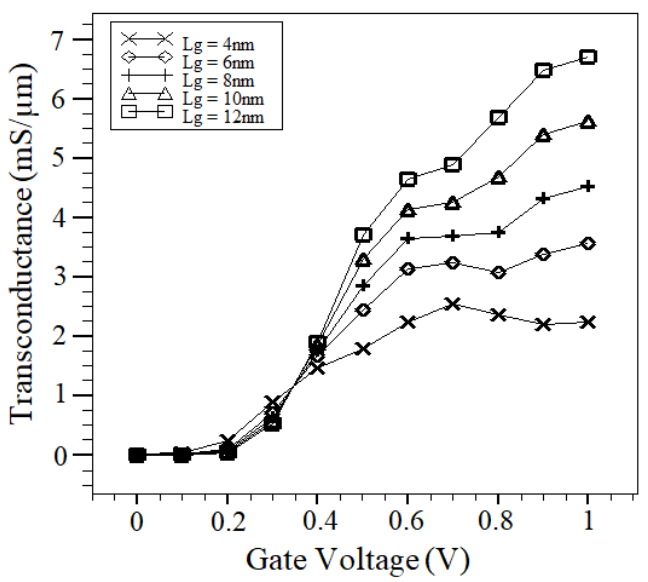

Fig. 9. Plot transconductance $\left(g_{m}\right)$ as a function of $V_{G}$

Based on the plot, it is observed that the $g_{m}$ of the JLSDGM device is decreased by approximately $58 \%$ as the $L_{g}$ is scaled down from $12 \mathrm{~nm}$ to $4 \mathrm{~nm}$. The smaller $L_{g}$ lessens the current flow due to much higher electric field in the channel. Since the $I_{D}$ is directly proportional with the $L_{g}$ (Fig. 5), any decrease in $V_{T H}$ would definitely lessen the $I_{D}$ as well as the $g_{m}$. Fig. 10 depicts the impact of $L_{g}$ variation on $g_{m}$ for JLSDGM device. Based on the plot, it can be concluded that the variation in $L_{g}$ is directly proportional with the $g_{m}$, indicating that as the $L_{g}$ is

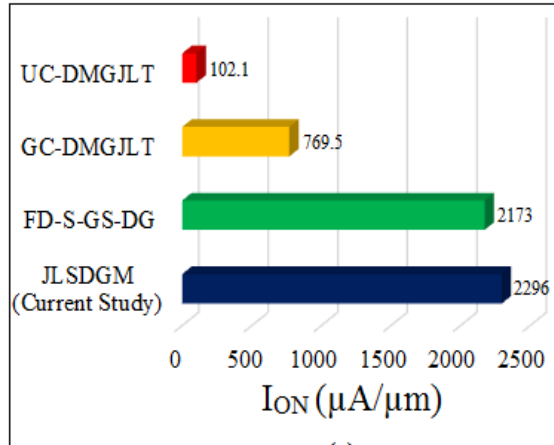

(a)

further scaled down from $12 \mathrm{~nm}$ to $4 \mathrm{~nm}$, the $g_{m}$ is constantly decreasing.

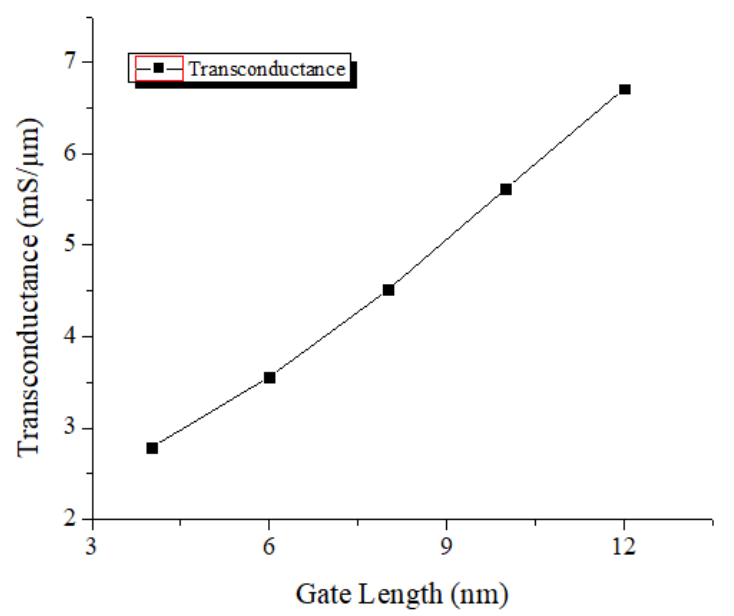

Fig. 10. Plot of transconductance versus gate length

It shows that the variation in $L_{g}$ do contribute a significant impact on the $g_{m}$ as the maximum transconductance, $g_{m}$ (max) is decreased over the reduction of $L_{g}$. The highest $g_{m}$ (max) of the JLSDGM device is measured at $6.71 \mathrm{mS} / \mu \mathrm{m}$ as the $L_{g}$ is scaled at $12 \mathrm{~nm}$. However, the device with $4 \mathrm{~nm}$ of $L_{g}$ still exhibits an acceptable $g_{m}(\max )$ value which is measured at $2.79 \mathrm{mS} / \mu \mathrm{m}$. Even with much smaller $L_{g}$, the JLSDGM device is still able to produce a quite high the $g_{m}(\max )$. This is mainly due to the bulk phenomenon effects where the carrier's absorption happens inside the entire part of the ultrathin body. An excellent transport efficiency of this JLSDGM device is portrayed by its high $g_{m}(\max )$ which is suitably applicable for analog based applications. The $I_{O N}$ and $g_{m}(\max )$ magnitude of the JLSDGM device with $8 \mathrm{~nm}$ gate length have been compared to different structures of double-gate transistor from previous studies $[50,51]$ as shown in Figure 11.

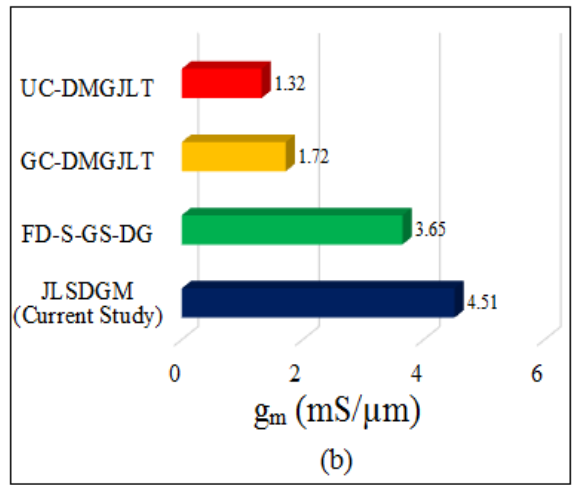

Fig. 11. Benchmark of Junctionless Strained Double Gate MOSFET (JLSDGM) with Uniform channel dual material gate junctionless transistor (UC-DMGJLT), Graded Channel Dual Material Gate Junctionless Transistor (GC-DMGJLT) and Fully

Depleted Strained Gate Stack Double gate (FD-S-GS-DG) for a) On-current $\left(I_{O N}\right)$; b) Max Transconductance $\left(g_{m}(\right.$ max $\left.)\right)$

Based on the comparison, it is shown that the current study (JLSDGM) has demonstrated the highest $I_{O N}$ and $g_{m}$ magnitudes compared to other double-gate structure technology. Both JLSDGM (current study) and FD-S-GS-DG devices have employed the strained channel to significantly boost the $I_{O N}$ and $g_{m}$ magnitudes. Large differences in $I_{O N}$ and $g_{m}(\max )$ magnitudes of JLSDGM (current study) and FD-S-GS-DG devices compared to UC-DMGJLT and GC-DMGJLT clearly imply that the presence of strained channel do form a high mobility field in the channel region of the double-gate device. Despite of being operated at low power supply $\left(V_{D D}=0.5 \mathrm{~V}\right)$, the JLSDGM device are still capable of producing a large $I_{O N}$ and $g_{m}(\max )$ magnitudes which makes it as one of the potential transistor's structure for low power and analog based applications. 


\section{RECOMMENDATIONS FOR FUTURE WORK}

From the observation of the results, it could be stated that the variation in gate length $\left(L_{g}\right)$ does contributes a significant impact on the JLSDGM's performance. Hence, the $L_{g}$ can be regarded as one of the critical design parameters that have to be controlled for tuning the JLSDGM's performance. Remarkable device properties like high $I_{O N}$, low $I_{O F F}$, high $I_{O N} / I_{O F F}$ ratio, low $S S$ and high $g_{m}$ at small scale of gate length implies that the JLSDGM device might be a potential transistor's configuration for future low power nano-scaled device. For future work, a comprehensive investigation on the impact of other design parameters besides $L_{g}$ (e.g. channel doping, S/D doping, work function and etc.) towards the JLSDGM's performance can be carried out. Besides, the investigation can be further extended by considering the analog and RF performance of the JLSDGM device. Furthermore, the DC and AC characteristics of the JLSDGM should be further enhanced by optimizing multiple design parameters simultaneously through various application of optimization approaches [52-58].

\section{CONCLUSION}

In summary, the design consideration and impact of gate length variation towards the drain current $\left(I_{D}\right)$, on-current $\left(I_{O N}\right)$, off-current $\left(I_{O F F}\right), I_{O N} / I_{O F F}$ ratio, subthreshold swing $(S S)$ and transconductance $\left(g_{m}\right)$ have been comprehensively discussed. The investigation has been conducted via 2-D process and device simulation that includes the drift diffusion (DD) model with simplified Boltzmann carrier statistics and the Lombardi CVT model which is combined with Shockley-Read-Hall (SRH). The results show that the variation in gate length $\left(L_{g}\right)$ does contribute a significant impact on the investigated JLSDGM's performance. The $I_{O N}$ of the JLSDGM device is decreased by approximately $46 \%$ as the $L_{g}$ is scaled down from $12 \mathrm{~nm}$ to $4 \mathrm{~nm}$. It is also shown that the $g_{m}$ of the JLSDGM device is decreased by approximately $58 \%$ as the $L_{g}$ is scaled down from $12 \mathrm{~nm}$ to $4 \mathrm{~nm}$. However, the JLSDGM device with $4 \mathrm{~nm}$ of $\mathrm{L}_{\mathrm{g}}$ still provides a remarkable $I_{O N}$ and $g_{m}(\max )$ in which both of them are measured at $1680 \mu \mathrm{A} / \mu \mathrm{m}$ and $2.79 \mathrm{mS} / \mu \mathrm{m}$ respectively. Such remarkable device characteristics indicate that the JLSDGM device could be a potential transistor's structure for low power based applications.

\section{ACKNOWLEDGMENT}

The authors would like to thank to the Ministry of Higher Education (MOHE) of Malaysia, Mybrain15 and Centre for Telecommunication Research and Innovation (CeTRI), Faculty of Electronics and Computer Engineering (FKEKK), Universiti Teknikal Malaysia Melaka (UTeM) for sponsoring this research works under research grants FRGS/1/2017/TK04/FKEKK-CeTRI/F00335.

\section{REFERENCES}

1. Y. C. Yeo, P. Ranade, T. J. King, and C. Hu, "Effects of high-k gate dielectric materials on metal and silicon gate workfunctions," IEEE Electron Device Letters, vol. 23, no. 6, pp. 342-344, 2002.

2. R. Chau, J. Brask, S. Datta, G. Dewey, M. Doczy, B. Doyle, J. Kavalieros, B. Jin, M. Metz, A. Majumdar, and M. Radosavljevic,
"Application of high-k gate dielectrics and metal gate electrodes to enable silicon and non-silicon logic nanotechnology," Microelectronic Engineering, vol. 80, pp. 1-6, 2005

3. R. Chau, S. Datta, M. Doczy, B. Doyle, J. Kavalieros, and M. Metz, "High-k/metal-gate stack and its MOSFET characteristics," IEEE Electron Device Letters, vol. 25, no. 6, pp. 408-410, 2004.

4. T. Ando, "Ultimate Scaling of High- $\kappa$ Gate Dielectrics: Higher- $\kappa$ or Interfacial Layer Scavenging?,” Materials, vol. 5, pp. 478-500, 2012.

5. N. M. Idris, A. Brown, J. Watling, and A. Asenov, "Simulation Study of Workfunction Variability in MOSFETs with Polycrystalline Metal Gates," Ultimate Integration on Silicon, no. March, pp. 165-168, 2010.

6. S.-H. Chen, W.-S. Liao, H.-C. Yang, S.-J. Wang, Y.-G. Liaw, H. Wang, H. Gu, and M.-C. Wang, "High-performance III-V MOSFET with nano-stacked high-k gate dielectric and 3D fin-shaped structure," Nanoscale Research Letters, vol. 7. p. 431, 2012.

7. I. Ferain, C. A. Colinge, and J. Colinge, "Multigate Transistors as the Future of Classical Metal-oxide-semiconductor Field-effect Transistors," Nature, vol. 479, pp. 310-316, 2011.

8. J. E. Suseno and R. Ismail, "Design of Double Gate Vertical MOSFET using Silicon On Insulator ( SOI ) Technology," International Journal of Nano Devices, Sensors and Systems (IJ-Nano), vol. 1, no. 1, pp. 34-38, 2012.

9. K. Boucart and A. M. Ionescu, "Double-Gate Tunnel FET With High-K Gate Dielectric," IEEE Transactions on Electron Devices, vol. 54, no. 7, pp. 4-12, 2008 .

10. K. E. Kaharudin, A. H. Hamidon, and F. Salehuddin, "Implementation of Taguchi Modeling for Higher Drive Current (ION) in Vertical DG-MOSFET Device," Journal of Telecommunication, Electronic and Computer Engineering, vol. 6, no. 2, pp. 11-18, 2014.

11. D. Hisamoto, W. Lee, J. Kedzierski, H. Takeuchi, K. Asano, C. Kuo, E. Anderson, T. King, J. Bokor, and C. Hu, "FinFET - A Self-Aligned Double-Gate MOSFET," IEEE Transactions on Electron Devices, vol. 47, no. 12, pp. 2320-2325, 2000.

12. M. A. Riyadi, J. E. Suseno, and R. Ismail, "The Future of Non-planar Nanoelectronics MOSFET Devices: A Review," Journal of Applied Sciences, vol. 10, no. 18, pp. 2137-2146, 2010.

13. M. Masahara, Y. Liu, S. Hosokawa, T. Matsukawa, K. Ishii, H. Tanoue, K. Sakamoto, T. Sekigawa, H. Yamauchi, S. Kanemaru, and E. Suzuki, "Ultrathin Channel Vertical DG MOSFET Fabricated by Using Ion-Bombardment-Retarded Etching," IEEE Transactions on Electron Devices, vol. 51, no. 12, pp. 2078-2085, Dec. 2004.

14. K. P. Pradhan, S. K. Mohapatra, P. K. Agarwal, P. K. Sahu, D. K. Behera, and J. Mishra, "Symmetric DG-MOSFET With Gate and Channel Engineering : A 2-D Simulation Study," Microelectronics and Solid State Electronics, vol. 2, no. 1, pp. 1-9, 2013.

15. J.-P. Colinge, "Multiple-gate SOI MOSFETs," Solid-State Electronics, vol. 48, no. 6, pp. 897-905, Jun. 2004.

16. Nader Shehata, Abdel-Rahman Gaber, Ahmed Naguib, Ayman E Selmy, Hossam Hassan, Ibrahim Shoeer, Omar Ahmadien, and Rewan Nabeel, "3D Multi-gate Transistors: Concept, Operation, and Fabrication," Jounal of Electrical Engineering, vol. 3, pp. 1-14, 2015.

17. S. Saurabh, M. J. Kumar, and S. Member, "Novel Attributes of a Dual Material Gate Nanoscale Tunnel Field Effect Transistor," IEEE Transactions on Electron Devices, vol. 58, no. February, pp. 404-410, 2011.

18. V. P. Trivedi, S. Member, and J. G. Fossum, "Scaling Fully Depleted SOI CMOS," IEEE Transactions on Electron Devices, vol. 50, no. 10, pp. 2095-2103, 2003.

19. K. E. Kaharudin, F. Salehuddin, A. S. M. Zain, M. N. I. A. Aziz, and I. Ahmad, "Application of Taguchi Method with the Interaction Test for Lower DIBL in WSix/TiO2 Channel Vertical Double Gate NMOS," ARPN Journal of Engineering and Applied Sciences, vol. 11, no. 11, pp. 7093-7103, 2016

20. B. Vandana, "Study of Floating Body Effect in SOI Technology," International Journal of Modern Engineering Research (IJMER), vol. 3 , no. June, pp. 1817-1824, 2013.

21. M. M. A. Hakim, L. Tan, A. Abuelgasim, K. Mallik, S. Connor, A Bousquet, C. H. De Groot, S. Member, S. Hall, and P. Ashburn, "Self-Aligned Silicidation of Surround Gate Vertical MOSFETs for Low Cost RF Applications," IEEE Transactions on Electron Devices, vol. 57, no. 12 , pp. $3318-3326,2010$. 
22. S. Dubey, P. K. Tiwari, and S. Jit, "On-current modeling of short-channel double-gate (DG) MOSFETs with a vertical Gaussian-like doping profile," Journal of Semiconductors, vol. 34, no. 5, p. 054001 , May 2013.

23. A. S. M. Zain, "Scaling and Variability in UltraThin Body Silicon on Insulator (UTB SOI) MOSFETs," University of Glasgow, 2013.

24. P. Choudhary and T. Kapoor, "Performance Based Comparative Analysis of MOS Structures at 32nm and 20nm Node," International Journal of Advanced Research in Computer and Communication Engineering, vol. 4, no. 3, pp. 137-141, 2015.

25. A. Chaudhry and M. J. Kumar, "Controlling Short-channel Effects in Deep Submicron SOI MOSFETs for Improved Reliability : A Review," IEEE Transaction on Device and Materials Reliability, vol. 4, no. March, pp. 99-109, 2004.

26. P. Olejarz, K. Park, S. MacNaughton, M. R. Dokmeci, and S. Sonkusale, "0.5 $\mu \mathrm{W}$ Sub-Threshold Operational Transconductance Amplifiers Using $0.15 \mu \mathrm{m}$ Fully Depleted Silicon-on-Insulator (FDSOI) Process," Journal of Low Power Electronics and Applications, vol. 2, pp. 155-167, 2012

27. M. N. I. A. Aziz, F. Salehuddin, A. S. M. Zain, and K. . Kaharudin, "Study of Electrical Characteristic for $50 \mathrm{~nm}$ and $10 \mathrm{~nm}$ SOI Body Thickness in MOSFET Device," Jurnal Teknologi, vol. 77, no. 21, pp. 109-115, 2015 .

28. R. Chau, S. Datta, M. Doczy, B. Doyle, B. Jin, J. Kavalieros, A. Majumdar, M. Metz, and M. Radosavljevic, "Benchmarking Nanotechnology for Logic Transistor Applications," IEEE Transactions on Nanotechnology, vol. 4, no. 2, pp. 153-158, 2005.

29. S. Saurabh and M. J. Kumar, "Impact of Strain on Drain Current and Threshold Voltage of Nanoscale Double Gate Tunnel Field Effect Transistor: Theoretical Investigation and Analysis," Japanese Journal of Applied Physics, vol. 48, no. 6, p. 064503, Jun. 2009.

30. H. W. Kim, B.-G. Park, J. H. Kim, and S. W. Kim, "Vertical type double gate tunnelling FETs with thin tunnel barrier," Electronics Letters, vol. 51, no. 9, pp. 718-720, 2015.

31. H. Valinajad, R. Hosseini, M. E. Akbari, A. Branch, and K. Branch, "Electrical Characteristics of Strained Double Gate," International Journal of Recent Research and Applied Studies, vol. 13, no. November, pp. 436-442, 2012.

32. L. Chang, S. Member, K. J. Yang, Y. Yeo, I. Polishchuk, T. King, S. Member, and $\mathrm{C}$. $\mathrm{Hu}$, "Direct-Tunneling Gate Leakage Current in Double-Gate and Ultrathin Body MOSFETs," IEEE Transactions on Electron Devices, vol. 49, no. 12, pp. 2288-2295, 2002.

33. T. K. Maiti, “An Explicit Surface-Potential Based Biaxial Strained-Si n-MOSFET Model for Circuit Simulation," Engineering, vol. 02, pp. 879-887, 2010

34. K. Chandrasekaran, X. Zhou, S. Member, S. Ben Chiah, W. Shangguan, G. H. See, L. K. Bera, N. Balasubramanian, S. C. Rustagi, and S. Ge, "Effect of Substrate Doping on the Capacitance - Voltage Characteristics of," IEEE Electron Device Letters, vol. 27, no. 1, pp. 62-64, 2006.

35. M. W. Akram, B. Ghosh, P. Bal, and P. Mondal, "P-type double gate junctionless tunnel field effect transistor," Journal of Semiconductors, vol. 35, no. 1, p. 014002, 2014.

36. Y. Chen, M. Mohamed, M. Jo, U. Ravaioli, and R. Xu, "Junctionless MOSFETs with laterally graded-doping channel for analog/RF applications," Journal of Computational Electronics, vol. 12, no. 4, pp. 757-764, 2013.

37. M. Wu, X. Jin, H.-I. Kwon, R. Chuai, X. Liu, and J.-H. Lee, "The Optimal Design of Junctionless Transistors with Double-Gate Structure for reducing the Effect of Band-to-Band Tunneling," JSTS:Journal of Semiconductor Technology and Science, vol. 13, no. 3, pp. 245-251, Jun. 2013.

38. A. K. Gupta, A. Kumar, and A. K. Jaiswal, "To Study and Characterisation of N N + N Nanowire Transistor ( Junctionless ) using 2D ATLAS Simulator," International Journal of Current Engineering and Technology, vol. 4, no. 3, pp. 2203-2206, 2014.

39. R. T. Doria, M. A. Pavanello, R. D. Trevisoli, M. De Souza, C. W. Lee, I. Ferain, N. D. Akhavan, R. Yan, P. Razavi, R. Yu, A. Kranti, and J. P. Colinge, "Junctionless multiple-gate transistors for analog applications," IEEE Transactions on Electron Devices, vol. 58, no. 8, pp. 2511-2519, 2011.

40. J. P. Colinge, A. Kranti, R. Yan, I. Ferain, D. Akhavan, P. Razavi, C. W. Lee, R. Yu, and C. A. Colinge, "A Simulation Comparison between Junctionless and Inversion-Mode MuGFETs.," ECS Transactions, vol. 35, no. 5, pp. 63-73, 2011.

41. J. P. Colinge, C. W. Lee, A. Afzalian, N. D. Akhavan, R. Yan, I. Ferain, P. Razavi, B. O’Neill, A. Blake, M. White, A. M. Kelleher, B.
McCarthy, and R. Murphy, "Nanowire transistors without junctions," Nature Nanotechnology, vol. 5, no. 3, pp. 225-229, 2010.

42. A. K. and R. Y. J. P. Colinge, C. W. Lee, N. Dehdashti Akhavan, R. Yan, I. Ferain, P. Razavi, Semiconductor-On-Insulator Materials for Nanoelectronics Applications, no. March 2015. Springer, 2011.

43. A. Kamath, X. Chen, N. Shen, X. Li, N. Singh, G. Q. Lo, and D.-L. Kwong, "Junctionless CMOS Transistors with Independent DoubleGates," International Journal of Information and Electronics Engineering, vol. 3, no. 1, pp. 13-15, 2013.

44. Z. Hong, A. Bodkhe, and S. Tzeng, "Method for Forming A Low Resistivity Tungsten Silicide Layer for Metal Gate Stack Applications," 2014.

45. K. E. Kaharudin, F. Salehuddin, N. Soin, A. S. M. Zain, M. N. I. A. Aziz, and I. Ahmad, "Electrical Characterization of Different High-k Dielectrics with Tungsten Silicide in Vertical Double Gate NMOS Structure," ARPN Journal of Engineering and Applied Sciences, vol. 11, no. 21 , pp. 12328-12335, 2016.

46. Silvaco, "Silvaco ATLAS manual Device Simulation Software," 2006.

47. K. E. Kaharudin, F. Salehuddin, A. S. M. Zain, and M. N. I. A. Aziz, "Optimization of Process Parameter Variations on Leakage Current in Silicon-on-insulator Vertical Double Gate Mosfet Device," Journal of Mechanical Engineering and Sciences (JMES), vol. 9, no. December, pp. 1614-1627, 2015.

48. F. A. Rezali, M. Mazhar, N. Aida, F. Othman, and S. W. Muhamad, "Performance and device design based on geometry and process considerations for 14 / 16 - nm FinFETs stress engineering," IEEE Transactions on Electron Devices, vol. 63, no. 3, pp. 974-981, 2016.

49. S. Sharma, P. Rahul, P. Aditya, and K. P. Vijendra, "An Analysis of Device Characteristics of Strained N-Channel MOSFET," SSRG International Journal of Electronics and Communication Engineering, vol. 3, no. 8, pp. 87-90, 2016.

50. K. P. Pradhan, S. K. Mohapatra, P. K. Sahu, and S. Parija, "Impact of Strain on Fully Depleted Strained Gate Stack Double Gate MOSFET : A Simulation Study Schematic structure of FD-S-GS-Double Gate N-MOSFET," ECTI Transactions on Electrical Engineering, ELectronics and Communications, vol. 13, no. 2, pp. 54-57, 2015.

51. V. Pathak and G. Saini, "A Graded Channel Dual-Material Gate Junctionless MOSFET for Analog Applications," Procedia Computer Science, vol. 125, pp. 825-831, 2018.

52. K. E. Kaharudin, F. Salehuddin, and A. S. M. Zain, "Optimization of Electrical Properties in TiO2 / WSix-based Vertical DG-MOSFET using Taguchi-based GRA with ANN," Journal of Telecommunication, Electronic and Computer Engineering, vol. 10, no. 1, pp. 69-76, 2018.

53. K. E. Kaharudin, F. Salehuddin, A. S. M. Zain, and M. N. I. A. Aziz, "Comparison of Taguchi Method and Central Composite Design for Optimizing Process Parameters in Vertical Double Gate MOSFET,' ARPN Journal of Engineering and Applied Sciences, vol. 12, no. 19, pp 5578-5590, 2017.

54. K. E. Kaharudin, F. Salehuddin, A. S. . Zain, and M. N. I. . Aziz, "Application of Taguchi-based Grey Fuzzy Logic for Simultaneous Optimization in TiO2/WSix-based Vertical double-gate MOSFET," Journal of Telecommunication, Electronic and Computer Engineering, vol. 9, no. 2-13, pp. 23-28, 2017.

55. K. E. Kaharudin, F. Salehuddin, A. S. M. Zain, M. N. I. A. Aziz, Z. Manap, N. A. A. Salam, and W. H. M. Saad, "Multi-response optimization in vertical double gate PMOS device using Taguchi method and grey relational analysis," in IEEE International Conference on Semiconductor Electronics (ICSE), 2016, pp. 64-68.

56. K. E. Kaharudin, F. Salehuddin, A. S. M. Zain, and M. N. I. A. Aziz, "Impact of Different Dose, Energy and Tilt Angle in Source / Drain Implantation for Vertical Double Gate PMOS Device," Journal of Telecommunication, Electronic and Computer Engineering, vol. 8, no. 5, pp. 23-28, 2016.

57. R. Norani, "A Dynamic Simulation on Single Gate Junctionless Field Effect Transistor Based on Genetic Algorithm," Advances in Computer Science: an International Journal, vol. 3, no. 5, pp. 140-145, 2014.

58. H. Ramakrishnan, "Variability: Analysis and Impact on Circuit Response," 2009. 


\section{AUTHORS PROFILE}

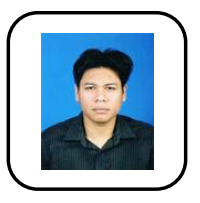

K. E. Kaharudin received Ph.D in Electronic Engineering and M. Eng degree in Computer Engineering from Technical University of Malaysia Melaka (UTeM), in 2017 and 2013 respectively. His Ph.D project focused on the process optimization of vertical double gate MOSFET. His research's interests include CMOS design, microelectronics, semiconductors, engineering optimization and artificial intelligence. Recently, his efforts emphasize on the simulation design of Junctionless MOSFET, silicon-on-insulator (SOI) MOSFET, high- $k /$ metal-gate stack technology, design of experiment (DoE) and predictive analytics.

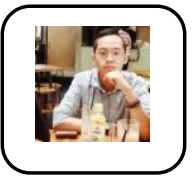

Ameer F. Roslan received B.Sc. Degree in Telecommunication Engineering from Technical University of Malaysia Melaka (UTeM), in 2017. He is currently pursuing his M.Sc degree in electronics and doing research on the DG-FinFET device at Centre for Telecommunication Research and Innovation, Faculty of Electronics and Computer Engineering, Technical University of Malaysia Melaka (UTeM). His research interest includes the DG-FinFET architecture and statistical analysis.

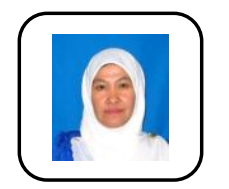

F. Salehuddin received the B.Eng. degree in electrical engineering (Communication) from Universiti Teknologi Mara (UiTM), Malaysia in 2001 and the M.Sc. degree in Electrical, Electronic and System Engineering from Universiti Kebangsaan Malaysia, in 2003. She received the $\mathrm{Ph} . \mathrm{D}$. degree in Microelectronics Engineering from Universiti Tenaga Nasional (UNITEN), Malaysia in 2012. She joined Universiti Teknikal Malaysia Melaka (UTeM) in December 2001 as a tutor and is currently a senior lecturer at Faculty of Electronic and Computer Engineering (FKEKK), UTeM. Her research interest includes process and device simulation of nanoscale MOSFETs device, advanced CMOS design, optimization approach (DOE) and process parameter variability.

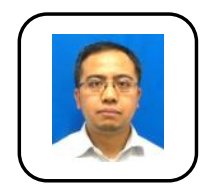

Z. A. F. M. Napiah received the B.Eng. degree in electrical engineering and the M.Eng. degree in Microelectronic from Universiti Teknologi Malaysia (UTM). He received the Ph.D. degree in Microelectronics from Kanawa University, Japan. He is currently a senior lecturer at Faculty of Electronic and Computer Engineering (FKEKK), UTeM. His research interest includes process and device simulation of MOSFET device, advanced CMOS design and CMOS based Photodetector and Photoreceiver.

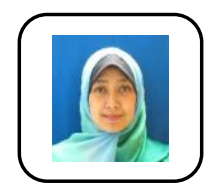

A. S. M. Zain received the B.Eng degree in electrical, Electronic and System engineering and $\mathrm{M}$. Sc in Microelectronics from National University of Malaysia (UKM), Malaysia She received the Ph.D. degree in Nanoelectronics Engineering from University of Glasgow, UK in 2013. She is currently working as a senior lecturer at Faculty of Electronic and Computer Engineering (FKEKK), Universiti Teknikal Malaysia Melaka (UTeM). Her research interest includes Nanoscale Device Design and Simulation, Variability and Reliability of Emerging Technology Devices, IC Design for Biomedical Applications. 